\title{
Student Daily Practices Identification Algorithm for Mobile Learning Application
}

\author{
Neculai Ivascu \\ School of Computing and Information Systems \\ Athabasca University \\ Athabasca, Canada \\ e-mail: claservco@yahoo.com
}

\author{
Maiga Chang \\ School of Computing and Information Systems \\ Athabasca University \\ Athabasca, Canada \\ e-mail: maiga.chang@gmail.com
}

\author{
Kristin Garn \\ CEO \& Research Department \\ Mathtoons Media \\ Kelowna, Canada \\ e-mail: kristin@mathtoons.com
}

\begin{abstract}
The purpose of this study is to introduce a daily student practice identification algorithm that is capable of discovering if a quiz is good for the student as daily practice so the student can practice what he/she is not good enough and sharpen his or her skill and knowledge. The psychometric parameters of each question are studied in terms of item difficulty, item discrimination and distractor analysis. In order to customize student practice, the system is able to suggest the daily practice according to his/her test knowledge level and proposed goals. The algorithm is designed to be used by Mathtoons Media's mobile application Practi to help students improve their school performance.
\end{abstract}

Keywords-multiple choice items, mobile app, quiz, classification, scaled score, context sensitive component

\section{INTRODUCTION}

The use of algorithms to monitor learning progress contributes to skill growth and provides good continuous feedback on the effectiveness of classroom instruction. Furthermore, it is a way to measure each student's progress toward personal goals in conformity with the principles for Fair Student Assessment Practices for Education [1]. To be effective in building skills, practice must be personalized, matching with the student's abilities.

The proposed study examines question and quiz items and suggests a classification algorithm that can be used to analyze the collected mobile app's usage data and measure the difficulty of a practice for the user. The participation of each particular question element is analyzed and quantified in terms of item difficulty, item discrimination and distractor analysis. According to [2], the Kuder-Richardson formula (KR20) is introduced to measure quiz reliability in addition to the student score analysis as a quantification of the quality of the quiz as a whole. Thus the proposed Scaled Score Calculation Algorithm is able to measure students' performance effectively, and reveal if necessary quiz improvements are needed when questions of low difficulty index or nonfunctional distractors are found.

The Daily Practice Suggestion Algorithm is then capable of choosing daily quests based on the user's performance and confidence level for the practice as well as the time decay factor. By simply setting the value of a priority variable, the algorithm automatically reacts to the individuals' knowledge deficiencies and suggests practice to sharpen specific skills.

\section{DEVELOPMENT OF KNOWLEDGE MEASUREMENT FOR MULTIPLE CHOICE ITEMS}

Norm-referenced tests are extensively used to evaluate student achievements since they provide objective measurement and permit comparison between individuals. They are often associated with standardized tests, reporting performance in terms of relative position in a normative group. The main characteristic of the norm-referenced quizzes is that student's achievement is awarded with reference to the performance of others within the specified group of interest [3]. When norm-referenced quizzes are developed, it is important to conduct item and quiz analyses to evaluate the difficulty and the discriminatory power of each item and the quality of the test as a whole [4].

The multiple-choice item format are often described as the most flexible and efficient item format for all types of tests, being suitable to a wide range of content and difficulty areas $[5,6,7]$. A well designed multiple-choice can test all the level of Bloom's taxonomy of cognitive objects: evaluation, synthesis, analysis, application, comprehension and knowledge [8]. Since it counts only the correct responses and the scoring is done by comparing students' answers with the scoring key without instructor intervention, the use of multiple-choice items produces highly objective results.

\section{DATA COLLECTING AND SCORING}

To evaluate the psychometric properties of the knowledge measurements, question level and quiz level analysis are conducted. Domain values for each of the variables are defined and a scaled score is calculated to indicate the student's location within the distribution of scores. The analytics used are [2]:

- Question difficulty index: The question difficulty index is the proportion of examinees who respond to an item correctly; 
- Distractors analysis: Distractors are classified as the incorrect answers in a multiple-choice question. A distractor used at a rate of 0.0 to 0.19 of the normal percentage indicates that it should be replaced from the pool of possible answers;

- Quiz reliability (KR20): The Kuder-Richardson formula is used to calculate the internal test consistency. The value of KR20 range from 0.0 to 1.0. A value of 0.60 or higher reveals that the quiz is a reasonably reliable instrument;

- Student scaled score: Scaled scores are calculated to unambiguously indicate the student's location within the cohort distribution of results and account the differences in difficulty across different items.

\section{A. Scaled Score Calculation Algorithm}

First, the algorithm checks whether or not a question is skipped by the student. If the question is not skipped, the algorithm updates the database with the number of students who answer the question and allocates points based on number of attempts, and if the hint was seen or not. Furthermore the program evaluates the item' psychometric parameters and more points are discriminative allocated based on these indicators. At the end, the program calculates the raw points and the student's scaled score.

\section{B. Daily Practice Suggestion Algorithm}

The Daily Practice Suggestion Algorithm is an efficient progress-monitoring and management tool of students' personalized daily practice. By simply setting the value of a priority variable, the algorithm automatically reacts to individual skill deficiencies and suggests student practice on specific skills. A question is considered difficult when its scaled score is lower than a chosen value. The highest rank into this variable is a skipped question.

When the student starts to use Practi, the algorithm checks if a list of practices for the student exists. If the practice list is empty, the algorithm calls Scaled Score Algorithm and builds a new list based on the results. The list is sorted based on the value of allocated variable priority. If the list is not empty, the algorithm reads the question from the list with the highest priority, checks the date when the question is accessed last time, and if the question isn't accessed the day before, it is then suggested to the student for daily practice.

If the question is taken by the student, its priority value will be altered to include the delayed in execution time. A question presented to the student which is successfully solved, is deleted from the list. The method runs until the last element is extracted from the list of practices, or until a user leaving event saves the rest of the list to the database.

\section{Plan OF Algorithms Evaluation AND USER ACCEPTANCE}

We propose a user-centric framework which links the objective system evaluation with user behavior through a series of perceptual and evaluative constructs.
For the proposed algorithms we consider the following accepting factors: user's perceived quality and usefulness of the given daily practices as well as user's behavioral intention in terms of whether or not the user is willing to do the given practice, and how frequently the user does the given practice daily. Empirical evaluations and investigative trade-offs between elements of user experience will be conducted. To ensure a robust measurement, questionnaires with a 5-point Likert scale will be used for each unidimensional concept. The study will be conducted online, on voluntary basis with students. The results will be analyzed to produce concept metrics.

\section{CONCLUSION}

Other than being only a student knowledge quantification tools, the proposed algorithms are able to analyze the effectiveness of each question in terms of item difficulty, item discrimination and distractor analysis. The KuderRichardson Formula (KR20) is used to compute the question's reliability. The Scaled Score Calculation Algorithm is able to measure students' performance effectively and reveal if necessary improvements are needed. The Daily Practice Suggestion Algorithm is able to react to individual skill deficiencies and suggests student practice on specific skills.

\section{ACKNOWLEDGMENT}

The authors wish to thank for the support of Athabasca University, NSERC, and industry partner Mathtoons Media.

\section{REFERENCES}

[1] Joint Advisory Committee, Principles for Fair Student Assessment Practices for Education in Canada, Ministry of Education, British Columbia, 2011. Retrieved February 2, 2015, from http://www.bced. gov.bc.ca /classroom_assessment/fairstudent.pdf

[2] Instructional Assessment Resources, Instructional Assessment Resources. Item Analysis, University of Texas at Austin, 2013. Retrieved February 2, 2015, from https:/www.utexas.edu/ academic/ $\mathrm{ctl} /$ assessment/iar/students/report/itemanalysis.php

[3] D. Ary, L. C. Jacobs, and A. Razavieh, Introduction to Research in Education, 6th Ed., Belmont, CA: Wadsworth, 2003.

[4] S. Matlock-Hetzel, "Basic Concepts in Item and Test Analysis," Annual Meeting of the Southwest Educational Research Association, Austin, TX, January 23-25, 1997. Retrieved February 2, 2015, from http://files.eric.ed.gov/fulltext/ED406441.pdf

[5] T. Kubiszyn and G. D. Borich, Educational Testing and Measurement, 6th Ed., Hoboken, NJ: John Wiley \& Sons Inc, 1999.

[6] T. M. Haladyna, S. M. Downing, and M.C. Rodriguez, "A review of multiple-choice item-writing guidelines for classroom assessment," Applied Measurement in Education, vol. 15 (3), pp. 309-334, 2002. Retrieved February 2, 2015, from http://valenciacollege.edu/faculty/ development/coursesResources/doc/SteveDowningHandout4.pdf

[7] A. J. Nitko and S. M. Brookhart, Educational assessments of students, 6th Ed., Englewood Cliffs, NJ: Prentice Hall, 2010.

[8] B.S. Bloom, Taxonomy of Educational Objectives, Hand book I: The Cognitive Domain, New York: David McKay Company Inc., 1956. 